NASA/TM-2001-211229

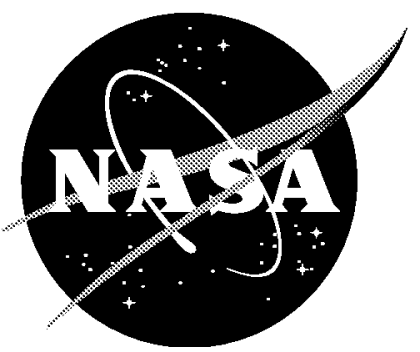

\title{
System Identification of a Vortex Lattice Aerodynamic Model
}

Jer-Nan Juang

Langley Research Center, Hampton, Virginia

Denis Kholodar and Earl H. Dowell

Duke University, Durham, North Carolina 


\section{The NASA STI Program Office ... in Profile}

Since its founding, NASA has been dedicated to the advancement of aeronautics and space science. The NASA Scientific and Technical Information (STI) Program Office plays a key part in helping NASA maintain this important role.

The NASA STI Program Office is operated by Langley Research Center, the lead center for NASA's scientific and technical information. The NASA STI Program Office provides access to the NASA STI Database, the largest collection of aeronautical and space science STI in the world. The Program Office is also NASA's institutional mechanism for disseminating the results of its research and development activities. These results are published by NASA in the NASA STI Report Series, which includes the following report types:

- TECHNICAL PUBLICATION. Reports of completed research or a major significant phase of research that present the results of NASA programs and include extensive data or theoretical analysis. Includes compilations of significant scientific and technical data and information deemed to be of continuing reference value. NASA counterpart of peer-reviewed formal professional papers, but having less stringent limitations on manuscript length and extent of graphic presentations.

- TECHNICAL MEMORANDUM. Scientific and technical findings that are preliminary or of specialized interest, e.g., quick release reports, working papers, and bibliographies that contain minimal annotation. Does not contain extensive analysis.

- CONTRACTOR REPORT. Scientific and technical findings by NASA-sponsored contractors and grantees.
- CONFERENCE PUBLICATION. Collected papers from scientific and technical conferences, symposia, seminars, or other meetings sponsored or co-sponsored by NASA.

- SPECIAL PUBLICATION. Scientific, technical, or historical information from NASA programs, projects, and missions, often concerned with subjects having substantial public interest.

- TECHNICAL TRANSLATION. Englishlanguage translations of foreign scientific and technical material pertinent to NASA's mission.

Specialized services that complement the STI Program Office's diverse offerings include creating custom thesauri, building customized databases, organizing and publishing research results ... even providing videos.

For more information about the NASA STI Program Office, see the following:

- Access the NASA STI Program Home Page at http://www.sti.nasa.gov

- E-mail your question via the Internet to help@sti.nasa.gov

- Fax your question to the NASA STI Help Desk at (301) 621-0134

- Phone the NASA STI Help Desk at (301) 621-0390

- Write to: NASA STI Help Desk NASA Center for AeroSpace Information 7121 Standard Drive Hanover, MD 21076-1320 
NASA/TM-2001-211229

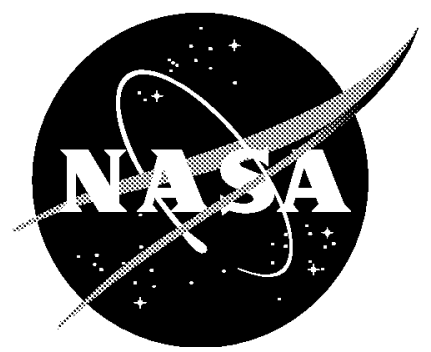

\section{System Identification of a Vortex Lattice Aerodynamic Model}

Jer-Nan Juang

Langley Research Center, Hampton, Virginia

Denis Kholodar and Earl H. Dowell

Duke University, Durham, North Carolina

National Aeronautics and

Space Administration

Langley Research Center

Hampton, Virginia 23681-2199 
Available from:

NASA Center for AeroSpace Information (CASI)

7121 Standard Drive

Hanover, MD 21076-1320

(301) 621-0390
National Technical Information Service (NTIS) 5285 Port Royal Road

Springfield, VA 22161-2171

(703) 605-6000 


\title{
System Identification of a \\ Vortex Lattice Aerodynamic Model
}

\author{
Jer-Nan Juang $^{1}$, Denis Kholodar ${ }^{2}$ and Earl H. Dowell ${ }^{3}$ \\ NASA Langley Research Center, Hampton, Virginia 23681-0001 \\ Duke University, Durham, North Carolina 27708-0300
}

\begin{abstract}
The state-space representation of an aerodynamic vortex lattice model is considered from a classical and system identification perspective. Using an aerodynamic vortex model as a numerical simulator of a wing tunnel experiment, both full state and limited state data or measurements are considered. Two possible approaches for system identification are presented and modal controllability and observability are also considered. The theory then is applied to the system identification of a flow over an aerodynamic delta wing and typical results are presented.
\end{abstract}

\footnotetext{
${ }^{1}$ Principal Scientist, Structural Dynamic Branch. Fellow AIAA

${ }^{2}$ Research Assistant, Department of Mechanical Engineering and Material Science.

${ }^{3}$ J. A. Jones Professor, Department of Mechanical Engineering and Material Science and Dean Emeritus, Pratt School of Engineering. Fellow AIAA. Director, Center for Nonlinear and Complex Systems.
} 


\section{Nomenclature}

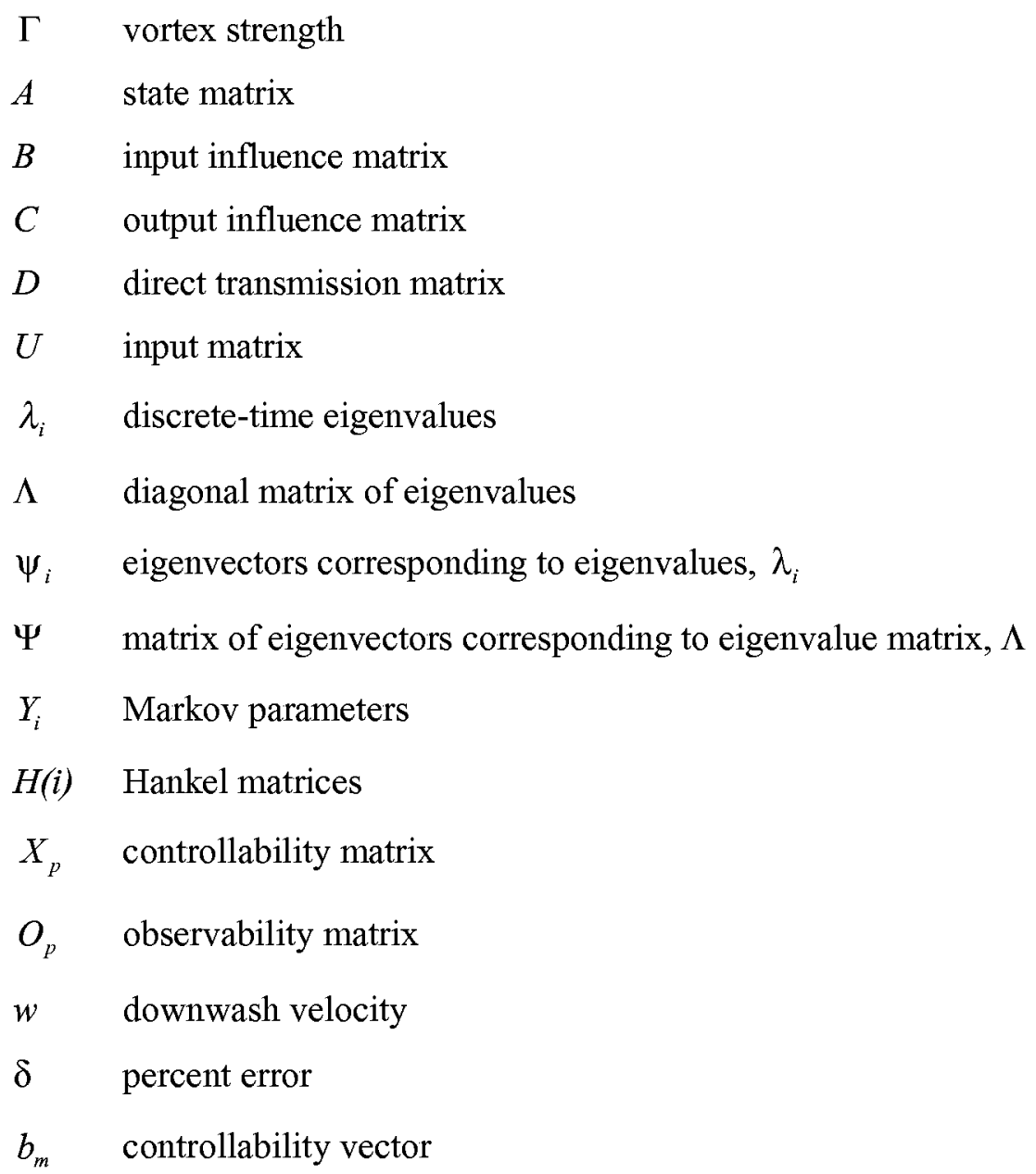




\section{Introduction}

In recent years, several studies have been conducted at Duke University of an aeroelastic delta wing system. In particular, reduced order unsteady aerodynamic models have been used to predict the flutter, limit cycle oscillations (LCO) and gust forced response of such a wing. For the case of incompressible, inviscid and irrotational flow, an unsteady vortex lattice method was used for time domain analyses. Moreover, once such a model was created, it was used to create a reduced order model (ROM) which allowed a reduction in the order of the vortex lattice code from a thousand degrees of freedom or more to the order of ten degrees of freedom while retaining essentially the same accuracy for the representation of fluid forces acting on a wing.

The development of such a vortex lattice model and a reduced order aerodynamic model based upon aerodynamic eigenmodes can be found in Ref. [1]. However, the representation of unsteady aerodynamic flow fields in terms of global aerodynamic modes can be developed in a variety of ways. In Ref. [2], the authors used Proper Orthogonal Decomposition modes and also a system identification model for a delta wing to obtain a reduced order model. The current work studies the system identification of a vortex lattice model in greater depth with a view to developing a methodology that can be used with wind tunnel experimental data. The vortex lattice model is used here as a test bed for a numerical experiment in a continuation of the work begun in Ref. [2].

\section{Delta Wing Vortex Model}

The flow about a cantilevered half-span delta wing is assumed to be incompressible, inviscid and irrotational. An unsteady vortex lattice method can be used to model this flow. A typical planar vortex lattice mesh for the three-dimensional flow over a wing is shown in Figure 1. The delta wing and the wake are divided into a number of vortex elements. Point vortices are placed on the wing and in the wake at the quarter chord of the elements. At the three-quarter chord of each plate element a collocation point is placed for the downwash and the velocity induced by the discrete vortices is set equal to the downwash (fluid vertical velocity on the wing) imposed by the prescribed 
delta wing motion or the gust field. Unsteady vorticity is shed into the wake and convected with the freestream velocity. The equations expressing these relationships are well described in Ref [1].

\section{State-Space Representation}

The relationship between a Vortex Lattice (VL) model and a state-space model developed by system identification methods will be established in this section. For simplicity, we begin with the assumption that full state information is given. This allows us to develop a formulation to gain basic insight before pursuing further development of the case where only partial state data are available.

\section{Full-State Data or Measurement}

The VL model can be described by ${ }^{1}$

$$
\tilde{A} \Gamma(k+1)+\tilde{B} \Gamma(k)=\tilde{D} u(k+1)
$$

where $\Gamma(k+1)$ is the strength vector of the vortex at the time step $k+1, \tilde{A}$ and $\tilde{B}$ are aerodynamic coefficient matrices, and $\tilde{D}$ is a transfer matrix for determining the relationship between the global vortex lattice mesh and the local vortex lattice mesh on the delta wing itself, and $u$ is the downwash vector. Expressions for $\tilde{A}, \tilde{B}$ and $\tilde{D}$ are given in Ref. [1]. Assume that there is a state-space model in the form

$$
\begin{aligned}
x(k+1) & =A x(k)+B u(k) \\
\Gamma(k) & =C x(k)+D u(k)
\end{aligned}
$$

where $A$ is the state matrix, $B$ is the input influence matrix, $C$ is the output influence matrix, and $D$ is the direct transmission matrix. Equation (2) gives the same map (relationship) as shown in Eq. (1) from $u$ to $\Gamma$. Is this assumption valid?

To answer this question, let us proceed as follows. Assuming that all states are measurable, the output matrix $C$ in Eq. (2) is square and invertible. Rewrite the bottom equation of Eq. (2) to become 


$$
x(k)=C^{-1} \Gamma(k)-C^{-1} D u(k)
$$

Substituting Eq. (3) into the top equation of Eq. (2) yields

$$
C^{-1} \Gamma(k+1)=A C^{-1} \Gamma(k)+\left[B-A C^{-1} D\right] u(k)+C^{-1} D u(k+1)
$$

Premultiplying Eq. (4) by $C$ produces

$$
\Gamma(k+1)=C A C^{-1} \Gamma(k)+\left[C B-C A C^{-1} D\right] u(k)+D u(k+1)
$$

Comparison of Eqs. (1) and (5) results in the following equalities

$$
\begin{aligned}
C A C^{-1} & =-\tilde{A}^{-1} \tilde{B} \\
D & =\tilde{A}^{-1} \tilde{D} \\
C B & =C A C^{-1} D=-\tilde{A}^{-1} \tilde{B} \tilde{A}^{-1} \tilde{D}
\end{aligned}
$$

From Eq. (6), it is clear that the state-space model is not unique in the sense that we have the freedom to choose $C$. Regardless of the choice of $C$, however, the eigenvalues of the VL model, i.e., eigenvalues of $-\tilde{A}^{-1} \tilde{B}$, are identical to those of $A$ (state matrix), as long as $C$ is invertible. For the case where $C$ is an identity matrix, i.e., $C=I$, Eq. (6) becomes

$$
\begin{aligned}
& A=-\tilde{A}^{-1} \tilde{B} \\
& D=\tilde{A}^{-1} \tilde{D} \\
& B=A D=-\tilde{A}^{-1} \tilde{B} \tilde{A}^{-1} \tilde{D}
\end{aligned}
$$

Thus, we have shown that the VL model, Eq. (1), has a state-space representation, Eq. (2), with system matrices, $A, B$, and $D$, uniquely determined by Eq. (7), assuming that the output matrix $C$ is an identity matrix. This state-space model may be used for model reduction and system identification.

Assume that we are given a (discrete time) sequence of

$$
\Gamma=\left[\begin{array}{llll}
\Gamma(1) & \Gamma(2) & \cdots & \Gamma(\ell)
\end{array}\right]
$$

and

$$
U=\left[\begin{array}{llll}
u(1) & u(2) & \cdots & u(\ell)
\end{array}\right]
$$

This may be a sequence of experimental data obtained from measurements or, as in the present case, numerical data from the vortex lattice model. One may use these sequences $\Gamma$ and $U$ to identify a set of system matrices, $A, B, C$, and $D$. If the identified $C$ is an 
invertible square matrix, the eigenvalues of the state matrix $A$ are, indeed, the eigenvalues of the VL model, i.e., the eigenvalues of $-\tilde{A}^{-1} \tilde{B}$. To make $C$ square and invertible requires that the number of measurements (rows) is identical to the number of states (columns). Furthermore, all rows of $U$ must be linearly independent for the system identification to be valid. If the rows of $U$ are not linearly independent, the matrix $B$ cannot be properly identified. Does this mean that the eigenvalues of $A$ may not be identified properly? The answer is "no". The eigenvalues of $A$ can be fully identified if some of the rows can sufficiently excite the system eigenvalues. See, for example, Ref. [3].

\section{Limited State Data or Measurements}

For simplicity, we have first developed the basic formulation based on a set of full state data or measurements. In practice, the spacial or temporal measurements may be extremely limited probably tens of spacial locations and/or time steps. For example, what if one is given only the first few elements of $\Gamma(\mathrm{k})$ for $k=1,2, \cdots, \ell$ generated by Eq. (1)?

Can we use this very limited number of elements to identify the eigenvalues of $-\tilde{A}^{-1} \tilde{B}$ ? One might intuitively conclude that it is very difficult, if not impossible. But, in fact, some progress can be mode as follows.

Let Eq. (1) be transformed to a new form in terms of modal coordinates, i.e.,

$$
\Gamma_{m}(k+1)+\Lambda \Gamma_{m}(k)=\tilde{D}_{m} u(k+1)
$$

where

$$
\begin{aligned}
\Lambda & =\Psi^{-1}\left[\tilde{A}^{-1} \tilde{B}\right] \Psi=\operatorname{diag}\left[\begin{array}{llll}
\lambda_{1} & \lambda_{2} & \cdots & \lambda_{n}
\end{array}\right] \\
\Gamma_{m}(k) & =\Psi^{-1} \Gamma(k) \\
\tilde{D}_{m} & =\Psi^{-1} \tilde{A}^{-1} \tilde{D}
\end{aligned}
$$

The quantities $\lambda_{1} \lambda_{2} \quad \cdots \quad \lambda_{n}$ are the eigenvalues of the VL model, and $\psi_{1} \quad \psi_{2} \quad \cdots \quad \psi_{n}$ are the corresponding eigenvectors that form the matrix $\Psi$. Assume that we have only one input, i.e., $u(k+1)$ in Eq. (10) is a scalar. All the modes will be excited if the column vector $\tilde{D}_{m}$ has no zero elements in it, and $u(k+1)$ is 
nonzero. If some elements of $\tilde{D}_{m}$ are zero, the corresponding eigenvectors will not be excited. Other inputs may be added to excite such modes, of course. The elements of $\tilde{D}_{m}$ with relatively larger magnitude will excite the corresponding eigenmode to have a larger response. Assume that we have excited all the modes by properly choosing some input locations and their excitation signal, i.e., none of the columns in

$$
\Gamma_{m}=\left[\begin{array}{llll}
\Gamma_{m}(1) & \Gamma_{m}(2) & \cdots & \Gamma_{m}(\ell)
\end{array}\right]
$$

is a zero vector. The output data or measurements will become

$$
\Gamma=\left[\begin{array}{llll}
\Gamma(1) & \Gamma(2) & \cdots & \Gamma(\ell)
\end{array}\right]=\Psi\left[\begin{array}{llll}
\Gamma_{m}(1) & \Gamma_{m}(2) & \cdots & \Gamma_{m}(\ell)
\end{array}\right]
$$

If $\Psi$ is invertible, each row of $\Gamma$ should include all information of the VL eigenvalues and eigenvectors. In theory, one should be able to extract the information of eigenvalues from one row of $\Gamma$ unless there exists some repeated eigenvalues.

Let us rewrite Eq. (1) to become

$$
\begin{aligned}
\Gamma(k+1) & =\hat{A} \Gamma(k)+\hat{D} u(k+1) \\
y(k) & =\hat{C} \Gamma(k)
\end{aligned}
$$

where

$$
\begin{aligned}
& \hat{A}=-\tilde{A}^{-1} \tilde{B} \\
& \hat{D}=\tilde{A}^{-1} \tilde{D}
\end{aligned}
$$

and $\hat{C}$ is an $m \times n$ matrix with $m<n$. Each row of $\hat{C}$ could have a unity at one location and zeros at all other locations so that $y(k)$ includes only the desired elements in $\Gamma(k)$. From Eq. (11), the following matrix equation can be easily derived, i.e.,

$$
\left[\begin{array}{c}
y(k) \\
y(k+1) \\
\vdots \\
y(k+p-1)
\end{array}\right]=\left[\begin{array}{c}
\hat{C} \\
\hat{C} \hat{A} \\
\vdots \\
\hat{C} \hat{A}^{p-1}
\end{array}\right] \Gamma(k)+\left[\begin{array}{cccc}
0 & 0 & 0 & 0 \\
0 & \hat{C D} & 0 & 0 \\
\vdots & \vdots & \ddots & \vdots \\
0 & \hat{C A^{p-2}} \hat{D} & \hat{C A} \hat{D} & \hat{C D}
\end{array}\right]\left[\begin{array}{c}
u(k) \\
u(k+1) \\
\vdots \\
u(k+p-1)
\end{array}\right]
$$

or

$$
y_{p}(k)=\Theta_{p} \Gamma(k)+\Upsilon_{p} u_{p}(k)
$$

where 


$$
\begin{gathered}
y_{p}(k)=\left[\begin{array}{c}
y(k) \\
y(k+1) \\
\vdots \\
y(k+p-1)
\end{array}\right] ; \quad u_{p}(k)=\left[\begin{array}{c}
u(k) \\
u(k+1) \\
\vdots \\
u(k+p-1)
\end{array}\right] ; \\
\Theta_{p}=\left[\begin{array}{c}
\hat{C} \\
\hat{C A} \\
\vdots \\
\hat{C A^{p-1}}
\end{array}\right] ; \quad \Upsilon_{p}=\left[\begin{array}{cccc}
0 & 0 & 0 & 0 \\
0 & \hat{C D} & 0 & 0 \\
\vdots & \vdots & \ddots & \vdots \\
0 & \hat{C A^{p-2}} \hat{D} & \hat{C A D} & \hat{C D}
\end{array}\right]
\end{gathered}
$$

where $p$ is an integer that must be chosen to make $\Theta_{p}$ a square matrix of $p m \times n$ with $p m=n$ and $m$ is the number of outputs. There is a great chance that such an integer $p$ does not exist. We will discuss this case later. The quantity $\Upsilon_{p}$ is a $p m \times p r$ matrix with $r$ being the number of inputs.

The quantity $y_{p}(k)$ is a $p m \times 1$ column vector stacking together the $m \times 1$ vectors, $y(k), y(k+1), \ldots, y(k+p-1)$ from different time steps, where $m$ is the number of outputs. Similarly, the quantity $u_{p}(k)$ is a $p r \times 1$ column vector consisting of $r \times 1$ vectors, $u(k)$, $u(k+1), \ldots, u(k+p-1)$, where $r$ is the number of outputs. Solving Eq. (13) for $\Gamma(k)$ yields

$$
\Gamma(k)=\Theta_{p}^{-1} y_{p}(k)-\Theta_{p}^{-1} \Upsilon_{p} u_{p}(k)
$$

Substituting $\Gamma(k)$ and $\Gamma(k+1)$ from Eq. (14) into Eq. (11) and pre-multiplying the resultant equation by $\Theta_{p}$ produces

$$
y_{p}(k+1)=\Theta_{p} \hat{A} \Theta_{p}^{-1} y_{p}(k)-\Theta_{p} \hat{A} \Theta_{p}^{-1} \Upsilon_{p} u_{p}(k)+\Upsilon_{p} u_{p}(k+1)+\Theta_{p} \hat{D} u(k+1)
$$

Assume that the number of inputs is $r$, i.e., $u(k+1)$ is a $r \times 1$ vector. Define $\hat{\Upsilon}_{p}$ as

$$
\hat{\Upsilon}_{p}=\Upsilon_{p} \text { with the first } \gamma \text { zero columns replaced by } \Theta_{\mathrm{p}} \hat{D}
$$

Equation (15) becomes

$$
y_{p}(k+1)=\Theta_{p} \hat{A} \Theta_{p}^{-1} y_{p}(k)-\Theta_{p} \hat{A} \Theta_{p}^{-1} \Upsilon_{p} u_{p}(k)+\hat{\Upsilon}_{p} u_{p}(k+1)
$$

For easy reference, let us call Eq. (17) the Generalized Vortex Lattice (GVL) model. This equation has a state-space representation similar to Eq. (2), i.e., 


$$
\begin{aligned}
x(k+1) & =A x(k)+B_{p} u_{p}(k) \\
y_{p}(k) & =C_{p} x(k)+D_{p} u_{p}(k)
\end{aligned}
$$

where

$$
\begin{aligned}
& A=\Theta_{p} \hat{A} \Theta_{p}^{-1} \\
& B_{p}=\Theta_{p} \hat{A} \Theta_{p}^{-1}\left[\hat{\Upsilon}_{p}-\Upsilon_{p}\right] \\
& C_{p}=I_{p m \times p m} \\
& D_{p}=\hat{\Upsilon}_{p}
\end{aligned}
$$

The output matrix $C_{p}$ is chosen to be an identity matrix. Equation (18) is a state-space model representing the map from the input vector $u_{p}(k)$ of $p r \times 1$ to the output vector $y_{p}(k)$ of $p m \times 1$. Now what we are looking for is the map from input vector $u(k)$ of $r \times 1$ to the output vector $y(k)$ of $m \times 1$. Careful examination of $\hat{\Upsilon}_{p}$ as defined in Eq. (16) reveals that

$$
\hat{\Upsilon}_{p}-\Upsilon_{p}=\left[\begin{array}{ll}
\Theta_{\mathrm{p}} \hat{D} & 0_{p m \times(p-1) r}
\end{array}\right]
$$

where $0_{p m \times(p-1) r}$ is an $p m \times(p-1) r$ zero matrix. Equation (18) thus reduces to

$$
\begin{aligned}
x(k+1) & =A x(k)+B u(k) \\
y(k) & =C x(k)+D u(k)
\end{aligned}
$$

where

$$
\begin{aligned}
A & =\Theta_{p} \hat{A} \Theta_{p}^{-1} \\
B & =\Theta_{p} \hat{D} \\
C & =\left[\begin{array}{ll}
I_{m \times m} & 0_{m \times(p-1) m}
\end{array}\right] \\
D & =\text { First } m \text { rows of } \Theta_{p} \hat{D}
\end{aligned}
$$

The measurement or data output equation for $\mathrm{y}(\mathrm{k})$ is obtained by taking the first $m$ rows of $y_{p}(k)$ shown in Eq. (18) and noting that $I_{p m \times p m}$ is an identity matrix and $\hat{\Upsilon}_{p}$ is a special matrix, i.e., the upper right-hand entries are all zeros. The matrix $A$ is related to $\hat{A}$ 
by a similarity transformation such that they share the same eigenvalues. We thus conclude that the eigenvalues for the VL model can be obtained from the state-space model shown in Eq. (21). The fundamental assumption is that the matrix $\Theta_{p}$ must be invertible. The matrix $\Theta_{p}$ is commonly referred to as the observability matrix in the control field. If some of the eigenvalues of the VL model are not observable, then $\Theta_{p}$ will not be full rank and thus not invertible. Then the identified state-space matrix $A$ shown in Eq. (21) would not include the un-observable eigenvalues. The maximum rank of $\Theta_{p}$ is the number of states, $n$. Therefore, even though we have an oversized $p m \times n$ matrix $\Theta_{p}$ with $p m>n$, a good system identification technique should be able to identify a minimum-size matrix $A$ that would include only the observable and controllable VL eigenvalues and eigenvectors.

\section{System Identification}

Several methods may be used for system identification (see Ref. [3]). Each has its own disadvantages and advantages. Here two simple approaches are presented, (1) Generalized Vortex Lattice (GVL) model identification and (2) Eigensystem Realization Algorithm (ERA).

\section{GVL Model identification}

The GVL model identification begins with Eq. (17). Let us rewrite Eq. (17) to become

$$
y_{p}(k+1)=A y_{p}(k)-A \Upsilon_{p} u_{p}(k)+\hat{\Upsilon}_{p} u_{p}(k+1)
$$

where the state matrix $A$ is defined in Eq. (22). Let the time index $k$ run from $l$ to $\ell$. Equation (16) will then produce the following matrix equation.

$$
Y_{p}(2)=A Y_{p}(1)-A \Upsilon_{p} U_{p}(1)+\hat{\Upsilon}_{p} U_{p}(2)
$$

or 


$$
Y_{p}(2)=\left[\begin{array}{lll}
A & -A \Upsilon_{p} & \hat{\Upsilon}_{p}
\end{array}\right]\left[\begin{array}{c}
Y_{p}(1) \\
U_{p}(1) \\
U_{p}(2)
\end{array}\right]
$$

where $Y_{p}(1), Y_{p}(2), U_{p}(1)$, and $U_{p}(2)$ are defined by

$$
\begin{aligned}
Y_{p}(k) & =\left[\begin{array}{lllll}
y_{p}(k) & y_{p}(k+1) & \cdots & y_{p}(\ell-p+k-1)
\end{array}\right] \\
& =\left[\begin{array}{cccc}
y(k) & y(k+1) & \cdots & y(\ell-p+k-1) \\
y(k+1) & y(k+2) & \cdots & y(\ell-p+k) \\
\vdots & \vdots & \ddots & \vdots \\
y(k+p-1) & y(k+p) & \cdots & y(\ell+k-2)
\end{array}\right]
\end{aligned}
$$

and

$$
\begin{aligned}
U_{p}(k) & =\left[\begin{array}{llll}
u_{p}(k) & u_{p}(k+1) & \cdots & u_{p}(\ell+p+k-1)
\end{array}\right] \\
& =\left[\begin{array}{cccc}
u(k) & u(k+1) & \cdots & u(\ell-p+k-1) \\
u(k+1) & u(k+2) & \cdots & u(\ell-p+k) \\
\vdots & \vdots & \ddots & \vdots \\
u(k+p-1) & u(k+p) & \cdots & u(\ell+k-2)
\end{array}\right]
\end{aligned}
$$

with $k=1$ and 2. Note that $A$ is an $n \times n$ matrix, $A \Upsilon_{p}$ is an $n \times p r$ matrix, and $\hat{\Upsilon}_{p}$ is also an $n \times p r$ matrix. Correspondingly, $Y_{p}(1)$ and $Y_{p}(2)$ are both $p m \times(\ell-p)$ matrices, and $U_{p}(1)$ and $U_{p}(2)$ are both $p r \times(\ell-p)$ matrices.

In Eq. (24), we have three matrices, $A, A \Upsilon_{p}$, and $\Upsilon_{p}$, with a total of $n \times(n+2 p r)$ unknowns to be determined. The matrix $\left[\begin{array}{c}Y_{p}(1) \\ U_{p}(1) \\ U_{p}(2)\end{array}\right]$ is of the size $(n+2 p r) \times(\ell-p)$. If the data length $\ell$ is given such that $\ell-p \geq n+2 p r$, then the maximum rank of the matrix is $n+2 p r$ if all the rows are linearly independent. Unfortunately, the matrices $U_{p}(1)$ and $U_{p}(2)$ have all rows in common except the first row of $U_{p}(1)$ and the last row of $U_{p}(2)$. This means that the three matrices, $A, A \Upsilon_{p}$, and $\hat{\Upsilon}_{p}$ cannot be solved uniquely. To solve the state matrix uniquely, let us define 


$$
\hat{\Upsilon}=\left[\begin{array}{ll}
0_{n \times r} & -A \Upsilon_{p}
\end{array}\right]+\left[\begin{array}{ll}
\hat{\Upsilon}_{p} & 0_{n \times r}
\end{array}\right]
$$

Using Eq. (25), Eq. (24) can be rearranged to become

$$
Y_{p}(2)=\left[\begin{array}{ll}
A & \hat{\Upsilon}
\end{array}\right]\left[\begin{array}{c}
Y_{p}(1) \\
U_{p+1}(1)
\end{array}\right]
$$

The matrix $U_{p+1}(1)$ is the union of $U_{p}(1)$ and $U_{p}(2)$, i.e., the $U_{p}(1)$ plus the last $r$ row of $U_{p}(2)$. Now, assume that an input signal is generated such that all rows of $U_{p+1}(1)$ are linearly independent, and the resulting $Y_{p}(1)$ from the measurement also has linearly independent rows. Equation (26) can then be solved to yield

$$
\left[\begin{array}{cc}
A & \hat{\Upsilon}_{p}
\end{array}\right]=Y_{p}(2)\left[\begin{array}{c}
Y_{p}(1) \\
U_{p+1}(1)
\end{array}\right]^{\dagger}
$$

where $\uparrow$ means pseudo-inverse. This is a least-squares solution if $\left[\begin{array}{c}Y_{p}(1) \\ U_{p+1}(1)\end{array}\right]$ has more columns than rows.

Equation (27) provides the state matrix $A$ as shown in Eq. (22) which in turns produces the eigenvalues of the VL model. Furthermore, Eq. (27) also produces the input matrix $B$ shown in Eq. (22) that is the first $r$ columns of $\hat{\Upsilon}_{p}$ [see Eqs. (16) and (22)]. Finally, the direct transmission term $D$ is the first $m$ rows of $B$ [see Eq. (22)].

Equation (27) for system identification is straightforward and intuitive. Nevertheless, it does not work for the case where the data length is so short that $\left[\begin{array}{c}Y_{p}(1) \\ U_{p+1}(1)\end{array}\right]$ has more rows than columns, i.e., more spacial measurement points than time data steps. On the other hand, the state matrix A produced by Eq. (23) will, in general, not be a minimum realization in the sense that it is oversized. In other words, the integer $p$ that produces the row number of $Y_{p}(1)$ and $U_{p+1}(1)$ may be chosen larger than the number of states. 


\section{Eigensystem Realization Algorithm}

Another approach that produces a minimum-size model is based on the minimum realization theory. This approach uses a sequence of responses generated by a pulse input to the real system and then the computation and inversion of the system transfer functions is obtained from the input and output data (see Chapter 5 and 6 of Ref. [3]). Let $u_{i}(0)=1(i=1,2, \ldots, r)$ and $u_{i}(k)=0(k=1,2, \ldots)$ be substituted into Eq. (21). When the substitution is performed for each input element $u_{i}(0)=1(i=1,2, \ldots, r)$, the results can be assembled into a pulse response matrix $Y$ with dimension $m$ by $r$ as follows:

$$
Y_{0}=D, Y_{1}=C B, Y_{2}=C A B, \cdots, Y_{k}=C A^{k-1} B
$$

The constant matrices in the sequence are known as Markov parameters (See Ref. [3]).

System identification begins by forming the generalized Hankel matrix $H(0)$, composed of the Markov parameters

$$
H(0)=\left[\begin{array}{cccc}
Y_{1} & Y_{2} & \cdots & Y_{q} \\
Y_{2} & Y_{3} & \cdots & Y_{q+1} \\
\vdots & \vdots & \ddots & \vdots \\
Y_{p} & Y_{p+1} & \cdots & Y_{p+q-1}
\end{array}\right]
$$

The fundamental rule is that the Hankel matrix must be formed such that its rank is larger than the order of the system to be identified. In theory, the Hankel matrix $H(0)$ and the state-space model are related by

$$
\begin{aligned}
H(0) & =\left[\begin{array}{cccc}
Y_{1} & Y_{2} & \cdots & Y_{q} \\
Y_{2} & Y_{3} & \cdots & Y_{q+1} \\
\vdots & \vdots & \ddots & \vdots \\
Y_{p} & Y_{p+1} & \cdots & Y_{p+q-1}
\end{array}\right] \\
& =\left[\begin{array}{c}
C \\
C A \\
\vdots \\
C A^{p-1}
\end{array}\right]\left[\begin{array}{llll}
B & A B & \cdots & A^{q-1} B
\end{array}\right]
\end{aligned}
$$

To determine $A, B, C$, first decompose the matrix $H(0)$ by using singular value decomposition to yield 


$$
\begin{aligned}
H(0) & =U \sum V^{T}=\left[\begin{array}{ll}
U_{r} & U_{t}
\end{array}\right]\left[\begin{array}{cc}
\sum_{r} & 0 \\
0 & \sum_{t}
\end{array}\right]\left[\begin{array}{ll}
V_{r} & V_{t}
\end{array}\right]^{T} \\
& =U_{r} \sum_{r} V_{r}^{T}+U_{t} \Sigma_{t} V_{t}^{T} \\
& \approx U_{r} \sum_{r} V_{r}^{T} \\
& =\left[U_{r} \sum_{r}^{1 / 2}\right]\left[\sum_{r}^{1 / 2} V_{r}\right]
\end{aligned}
$$

where $U$ and $V$ are orthonormal matrices such that $U^{T} U=I$ and $V^{T} V=I$, and $\Sigma_{t}$ is a diagonal matrix containing the singular values that are negligible in comparison with those in the diagonal matrix $\Sigma_{r}$. Comparison of Eqs. (30) and (31) establishes the following equalities

$$
\begin{aligned}
& C=\text { First } m \text { rows of }\left[U_{r} \sum_{r}^{1 / 2}\right] \\
& B=\text { First } r \text { columns of }\left[\sum_{r}^{1 / 2} V_{r}\right]
\end{aligned}
$$

The equalities in Eq. (32) are not unique, but they are balanced because both share the matrix $\sum$ equally. To determine the state matrix $A$, another Hankel matrix must be formed, i.e.,

$$
H(1)=\left[\begin{array}{cccc}
Y_{2} & Y_{3} & \cdots & Y_{q+1} \\
Y_{3} & Y_{4} & \cdots & Y_{q+2} \\
\vdots & \vdots & \ddots & \vdots \\
Y_{p+1} & Y_{p+2} & \cdots & Y_{p+q}
\end{array}\right]
$$

This matrix is formed by deleting the first column of $H(0)$ and adding a new column at the end of the matrix. As a result, $H(l)$ has the following relationship with the system matrices $A, B$, and $C$

$$
\begin{aligned}
H(1) & =\left[\begin{array}{cccc}
Y_{2} & Y_{3} & \cdots & Y_{q+1} \\
Y_{3} & Y_{4} & \cdots & Y_{q+2} \\
\vdots & \vdots & \ddots & \vdots \\
Y_{p+1} & Y_{p+2} & \cdots & Y_{p+q}
\end{array}\right] \\
& =\left[\begin{array}{c}
C \\
C A \\
\vdots \\
C A^{p-1}
\end{array}\right] A\left[\begin{array}{llll}
B & A B & \cdots & A^{q-1} B
\end{array}\right]
\end{aligned}
$$


Substituting Eq. (31) into Eq. (34) thus yields

$$
\begin{aligned}
A & =\left[U_{r} \Sigma_{r}^{1 / 2}\right]^{\dagger} H(1)\left[\sum_{r}^{1 / 2} V_{r}\right]^{\dagger} \\
& =\Sigma_{r}^{-1 / 2} U_{r}^{T} H(1) V_{r}^{T} \Sigma_{r}^{-1 / 2}
\end{aligned}
$$

The symbol $\uparrow$ means pseudo-inverse. The orthonormal property of $U$ and $V$ shown in Eq. (31) has been used to derive Eq. (35).

Assume that the state matrix $A$ of order $n$ has a complete set of linearly independent eigenvectors $\psi_{1}, \psi_{2}, \cdots, \psi_{n}$ with corresponding eigenvalues $\lambda_{1}, \lambda_{2}, \cdots, \lambda_{n}$ which are not necessarily distinct. Define $\Lambda$ as the diagonal matrix of eigenvalues and $\Psi$ as the matrix of eigenvectors, i.e.,

$$
\Lambda=\left[\begin{array}{llll}
\lambda_{1} & & & \\
& \lambda_{2} & & \\
& & \ddots & \\
& & & \lambda_{n}
\end{array}\right]
$$

and

$$
\Psi=\left[\begin{array}{llll}
\psi_{1} & \psi_{2} & \cdots & \psi_{n}
\end{array}\right]
$$

The identified $A, B$, and $C$ can then be transformed to become $\Lambda, \Psi^{-1} B$, and $C \Psi$. The state-space model, Eq. (21), in modal coordinates, becomes

$$
\begin{aligned}
x_{m}(k+1) & =\Lambda x_{m}(k)+B_{m} u(k) \\
y(k) & =C_{m} x_{m}(k)+D u(k)
\end{aligned}
$$

where

$$
\begin{aligned}
\Lambda & =\Psi^{-1} A \Psi \\
x_{m}(k) & =\Psi^{-1} x(k) \\
B_{m} & =\Psi^{-1} B \\
C_{m} & =C \Psi
\end{aligned}
$$

The diagonal matrix $\Lambda$ contains the modal damping rates and the damped natural frequencies. The matrix $B_{m}=\Psi^{-1} B$ defines the initial modal amplitudes and the matrix $C_{m}=C \Psi$ the mode shapes at the sensor points. All the modal parameters of a dynamic system can thus be identified by the three matrices $\Lambda, \Psi^{-1} B$, and $C \Psi$. The desired modal damping rates and damped natural frequencies are simply the real and imaginary 
parts of the eigenvalues $\Lambda_{c}$, after transformation from the discrete-time domain to the continuous-time domain using the relation

$$
\Lambda_{c}=\frac{1}{\Delta t} \log (\Lambda)
$$

\section{Modal Controllability and Observability}

Equation (30) shows that the Hankel matrix $H(0)$ is formed by two matrices defined by

$$
\mathcal{O}_{p}=\left[\begin{array}{c}
C \\
C A \\
\vdots \\
C A^{p-1}
\end{array}\right]
$$

and

$$
\mathcal{C}_{q}=\left[\begin{array}{llll}
B & A B & \cdots & A^{q-1} B
\end{array}\right]
$$

The matrix $\mathcal{O}_{p}$ is commonly called the observability matrix whereas $\mathcal{C}_{q}$ is referred to as the controllability matrix. If the system possesses $n$ states, both $\mathcal{O}_{p}$ and $\mathcal{C}_{q}$ may have a maximum rank of $n$ assuming that the integers $p$ and $q$ are sufficiently large. Note that $\mathcal{O}_{p}$ and $\mathcal{C}_{q}$ may not share the same rank. If either $\mathcal{O}_{p}$ or $\mathcal{C}_{q}$ is short of rank $n$, then some of eigenvalues may not be identifiable. Let us elaborate on this statement by computing $\mathcal{O}_{p}$ and $\mathcal{C}_{q}$ in modal coordinates.

The matrices $\mathcal{O}_{p}$ and $\mathcal{C}_{q}$ in modal coordinates become

$$
\tilde{\mathcal{O}}_{p}=\left[\begin{array}{c}
C_{m} \\
C_{m} \Lambda \\
\vdots \\
C_{m} \Lambda^{p-1}
\end{array}\right]=\left[\begin{array}{c}
C \Psi \\
C \Psi\left[\Psi^{-1} A \Psi\right] \\
\vdots \\
C \Psi\left[\Psi^{-1} A \Psi\right]^{p-1}
\end{array}\right]=\mathcal{O}_{p} \Psi
$$

and 


$$
\begin{aligned}
& \tilde{\mathcal{C}_{q}}=\left[\begin{array}{llll}
B_{m} & \Lambda B_{m} & \cdots & \Lambda^{q-1} B_{m}
\end{array}\right] \\
& \left.=\left[\begin{array}{llll}
\Psi^{-1} B & {\left[\Psi^{-1} A \Psi\right] \Psi^{-1} B} & \cdots & {\left[\Psi^{-1} A \Psi\right.}
\end{array}\right]^{q-1} \Psi^{-1} B\right] \\
& =\Psi^{-1} \mathcal{C}_{q}
\end{aligned}
$$

The matrix $\tilde{\mathcal{O}}_{p}$ is the modal observability matrix whereas $\tilde{\mathcal{C}}_{q}$ is the modal controllability matrix. Both matrices are coordinate dependent. Nevertheless, the Hankel matrix $H(0)$ is coordinate invariant,

$$
H(0)=\tilde{\mathcal{O}}_{p} \tilde{\mathcal{C}}_{q}=\mathcal{O}_{p} \Psi \Psi^{-1} \mathcal{C}_{q}=\mathcal{O}_{p} \mathcal{C}_{q}
$$

There are $n$ columns in $\mathcal{O}_{p}$ and $n$ rows in $\mathcal{C}_{q}$.

Let $o_{i}$ be the $i$ th column of $\mathcal{O}_{p}$ and $c_{i}^{T}$ the $i$ th row of $\mathcal{C}_{q}$. The Hankel matrix $H(0)$ can be rewritten as

$$
H(0)=\tilde{\mathcal{O}}_{p} \tilde{\mathcal{C}}_{q}=\sum_{i=1}^{n} o_{i} c_{i}^{T}
$$

Both $o_{i}$ and $c_{i}^{T}$ are associated with the $i$ th eigenvalue $\lambda_{i}$. The matrix $H(0)$ is the sum of $n$ rectangular matrices formed by the product of $o_{i} c_{i}^{T}$ with $i=1, \ldots n$. Either $o_{i}$ or $c_{i}^{T}$ will have contribution to $H(0)$ only if it is not a null vector. For example, if the input actuators are located at the nodes of the $i$ th mode shapes, the row vector $c_{i}^{T}$ is null and thus its norm is zero. A similar statement is also true for the output sensors and the corresponding vector $o_{i}$. The degree of contribution of the system eigenvalue $\lambda_{i}$ to $H(0)$ is determined by the product of $o_{i} c_{i}^{T}$ which yields a $p m \times q r$ matrix. The norms $\left\|o_{i}\right\|$ and $\left\|c_{i}\right\|$ are commonly used to measure the degree of observability and controllability, respectively, for the eigenvalue $\lambda_{i}$. On the other hand, the product $\left\|o_{i}\right\|\left\|c_{i}\right\|$ is a measure of the contribution of $\lambda_{i}$ to $H(0)$. A relatively larger product $\left\|o_{i}\right\|\left\|c_{i}\right\|$ means easier identification of the eigenvalue $\lambda_{i}$ from the input and output data. Note that the input signal must be rich enough to excite the controllable and observable modes to be identified. 


\section{Numerical Example}

A system identification (SID) model for an unsteady aerodynamic flow has been created for several wing motions or gust excitations and corresponding aerodynamic responses. These models were derived from numerical simulations using a vortex lattice (VL) model for a delta wing with 55 vortex elements on the wing and 300 vortex elements in the wake (i.e. in Fig. $1, k m=k n=10, k m m=20$ ). In each case, the flow about the wing is excited by a certain type of prescribed downwash at the wing points, $w(t)$.

The numerical VL model produced vortex strengths at the grid points of the wing and in the wake, $\Gamma(t)$, and the corresponding pressure distribution on the wing, $p(t)$. These data were then used as input for the SID model.

The following excitations to the flow have been considered:

1) step angle of attack, $w(t)=$ const for $t>0$,

2) frozen (fixed with respect to the fluid fixed coordinates) sharp edge gust, $w(t$ $\mathrm{x} / v)=$ const for $(t-\mathrm{x} / v)>0$, where $v$ is the airfoil or flow velocity,

3) frozen gust of changing frequency, $w(t-\mathrm{x} / v)=$ const $\sin \left(\omega_{\max }(t-\mathrm{x} / v)^{2} / 2 T\right)$, which is sometimes called a swept gust, where the frequency of the sweep changes from zero to the maximal frequency, $\omega_{\max }$, within the period of the sweep, $T$.

4) random downwash ( $w$ at each grid point and at every time step is a random number),

5) frozen random gust ( $w$ at the first grid point [root leading edge element] is a random number at every new time step, that $w$ is then convected with the freestream velocity for the following panels during next steps).

The excitation cases $1,2,3$, and 5 are $x$ coordinate dependent only. The excitation signal at a specific location $x$ is constant along the axis $y$ as shown in Fig. 1. This implies that the excitation data matrices used for system identification are rank deficient, i.e., the rank is less than the number of inputs. The system matrices $A, B, C$, and $D$ thus identified by using these types of excitation would not be accurate, in particular the columns in the input matrix $B$ may not be independent. The number of independent columns in $B$ is equal to the rank of the excitation data matrix. 
On the other hand, the random excitation defined in case 4 is both $\mathrm{x}$ and y coordinate dependent. The excitation data matrix used for identifying the system matrices $A, B, C$, and $D$ is of full rank (i.e., the number of inputs) with the assumption that the excitation data length is equal to or longer than the number of inputs. The input matrix $B$ in particular would be accurately identified in theory for a controllable and observable system. All columns in B are linearly independent.

\section{Using a system identification model}

The ability of a SID model obtained from one of the excitations to predict the flow for another possible excitation was studied. The aformentioned five flow excitations were considered. Results were obtained for either vortex strength data or pressure data. Consider the vortex strength data case first. Using the time histories (1000 time steps) for the downwash and the vortex strengths, where the latter was generated by the VL model for the given downwash, a SID model was obtained. That is, the state-space matrices A, $\mathrm{B}, \mathrm{C}$, and D for this particular downwash were computed. Then this SID model was used to predict the vortex strength histories due to another downwash and the results compared with the corresponding vortex strength time histories obtained by the VL model itself for that downwash. To quantify the differences between the VL and SID outputs an error, $\delta$, defined as $\delta=100 \% / Q-y / / / Q /$ was used, where the norm $/ X /$ is defined to be the largest singular value of $X$. In this case, both $Q$ (VL vortex strengths) and $y$ (SID predicted vortex strengths) are $1000 \times 355$ rectangular matrices (1000 time steps and 355 degrees of freedom). The same method was also used for the pressure data. There $Q$ (VL pressure) and $y$ (SID predicted pressure) are $1000 \times 55$ (55 elements on the wing) matrices. These results are presented in Figures 2 and 3 and in Tables 1 and 2.

Consider Fig. 2. Here the percent errors are sorted based upon using the wing motion or gust excitation used to obtain a SID model, while the errors correspond to how well that SID model predicts the vortex strength (Fig 2a) and pressure (Fig. 2b) time histories for other excitations. A limit of 50\% is used for the vertical axis scale in order to show small errors better. When applied to the very same downwash, the SID models compute the output with the less than $1 \%$ error (see Ref. [2]). As seen in the figure for 
both the vortex strengths and pressure, the random excitation case is the best choice to obtain an SID model that will predict the behavior for any of the considered cases. In Fig $2 \mathrm{a}$ one can see that, if not asked to predict the very special case of a random downwash, three other models based on sharp edge, swept gust and frozen random excitation also work reasonably well.

The results in Figure 3 are sorted based upon the flow one is trying to predict with a SID model. For example, from the Fig. 3a one can conclude that to be able to predict the vortex strengths due to a frozen swept or frozen random gust, SID models obtained from any excitation case (except the step angle of attack excitation) would do a very good job, while for the prediction of vortex strengths due to a step angle of attack wing motion or frozen gust excitation any of considered SID models would perform well. Not surprisingly (after studying Fig.2), both Fig. 3a and 3b show that only a SID model obtained from random downwash data can predict the time history of aerodynamic flow due to the random downwash. The values of these errors are presented in the Tables 1 (vortex strengths) and 2 (pressure), where the rows of the tables relate to the Fig. 2 and columns to the Fig. 3 .

It is useful to consider the number of singular values kept in the SID models. The number following the wing motion/gust designation in the first column of the tables is the number of singular values retained in the identified SID model for that wing motion/gust. For the vortex strength data cases, the maximum number of singular values varies from 50 for the sharp edge gust to 300 for the random gust. This number is chosen such that the ratio of the largest singular value to the smallest one kept was $10^{12}$. For the vortex strength case, the total number of singular values is 300 (which, in fact, is equal to the total number of panels minus the number of panels on the wing). Thus, in the random gust case the signal is such that all the singular values are relatively high and close to each other magnitude.

\section{Lift}

As a global measure of the aerodynamic flow, consider the lift on the wing vs time. See Fig. 4. In Fig. 4a, $\mathrm{b}$ the lift time history for the motion of the wing due to the 
step angle of attack is presented. In Fig. 4c, $d$ the lift time history for the excitation by the frozen sharp edge gust is shown. (Each case is split into two figures to show in detail the predicted steady state lift.) The solid line is the "original" lift (obtained from the VL model). The left pointed and right pointed triangles represent lift time histories from SID models obtained from the step angle of attack motion and frozen sharp edge gust respectively, circles - frozen swept gust, squares - random gust, and diamonds - frozen random gust. (Note that the steady state lift due to either step angle of attack or sharp edge gust of the same amplitude is the same. That is why Fig. $4 \mathrm{~b}$ looks very much like Fig. 4d.) By comparing the graphs in Fig. 4 with either results in Fig $3 \mathrm{~b}$ or Table2, one can conclude that the error norm previously discussed does a good job of reflecting the difference between the "original" flow and those predicted by the identified models. However, the additional insight gained from the plots in Fig. 4 (see Fig. 4b and d) is that SID models from all excitations (but the swept gust) predict the steady state flow very well.

From the above numerical results, it was clearly shown that the system response may not be reproduced using an identified model from excitations other than the random gust or its own excitation. Note that only the random gust could identify an accurate model described by the system matrices $A, B, C$, and $D$ for all other inputs. Other kinds of excitation including the step angle, sharp edge, swept gust, and frozen random could only reproduce its own response, although some excitations such as swept gust may identify a model that gives a reasonable prediction for the responses produced by step angle, sharp edge, and frozen random. Nevertheless, none of the identified models produced by the step angle, sharp edge, swept gust, and frozen random could predict the response excited by the random gust. This is because the excitations other than the random gust did not excite all system modes in order to identify a state-space model to represent the system accurately. Recall that only in the case of random gust all the excitation (downwash) data are independent while in the four other cases only ten of them are independent (the downwash along the y coordinate is the same). Some modes can only be excited by independent downwash along the $\mathrm{x}$ coordinate as well as the $\mathrm{y}$ coordinate. 


\section{Modal Controllability and Observability}

The modal observability and controllability matrices (42) and (43) were computed for small numbers of outputs, $Y$, and inputs, $U$, respectively. The ranks of the controllability and observability matrices ranged between 63 and 91 depending on what output and input elements were used when building (42) and (43). For example, the rank of the controllability matrix in the case when there is only one input applied at the tip element of the wing (only the last column of the full input influence matrix, $B$, remains) was 91 . Remember the system posses $n=355$ states, the maximum rank can be as much as 355 . Therefore, because the ranks of the controllability and observability matrices are less than 355 , the system is may not be controllable and/or observable if only one input located at the tip element of the wing is used. Moreover, a controllability vector, $b_{m}$, (see section 5.1.1 of Ref. [3]) was computed. This vector is formed by the product of the inverse of the modal matrix with the eigenvectors of $A$ as its column vectors and a column of the input influence matrix $B$. It was previously shown (Ref. [3]) that if $b_{m}$ has a zero element, than the control force applied at that modal node cannot control this mode. This means that the control force is acting exactly at the node of the mode.

It was found that the modes corresponding to the dominant branch of the aerodynamic flow eigenvalues (for an earlier discussion of the eigenvalues see Ref. [2]) are the least controllable for the input located at the tip element of the wing. The components of the vector $b_{m}$ were scaled such that the largest component is 1. In Figure 5, the continuous time eigenvalues of the system are shown: in Fig. 5a those eigenvalues that have corresponding components of the controllability vector with values larger than 0.1 are noted by a cross, $\mathrm{x}$; in Fig. 5b similar results are shown for 0.01 . As one can see, even in the latter case the eigenvalues of the dominant branch are still not marked.

Values of all components of the controllability vector are shown in Figure 6a. The mode number is plotted along the horizontal axis, while the vertical axis shows the value of the components of the scaled vector $b_{m}$. The modes corresponding to the dominant branch of the eigenvalues are numbered from 1 to 25 (the first 10 of which correspond to the closest to zero circle), and as seen from Fig. 6a they are the least controllable. The 
same result persists if other locations of the input force are considered or indeed if the force applied at more than one location including all 55 vortex lattice panels on the wing.

An investigation of modal observability showed that the dominant modes are the most observable. Observability vector components are presented in Figure $6 \mathrm{~b}$ and the continuous time eigenvalues that correspond to modes with scaled observability vector larger than certain value are presented in Figure 7. In Fig. 7a, the components larger than 0.4 were crossed (note the two most right circles corresponding to first 11 modes are crossed) and in Fig. 7b, all of the components that are larger than 0.2 were marked. Unlike the controllablity case, the modes that correspond to the dominant branch are the most observable.

Qualitatively the same controllability and observability results were found when the identified system was obtained using considered excitations. Such results for the case of the random gust excitation are presented in Figure 8: the modes corresponding to the dominant branch are the least controllable (Fig 8a) and the most observable (Fig 8b).

When pressure data was used to obtain A, B, and C matrices, again the system was found to be neither controllable nor observable with only one input located at the tip of the delta wing, even though the scaled controllability and observability vectors look different in this case: these vectors for the random gust excitation are presented in Figure 9. Note, however, that the 55 modes that appear here do not correspond to those of the VL model or at least such a relation was not established.

Modal controllability provides a measure of how difficult it may be to excite the respective mode by the input(s). For simplicity without losing generality, let us consider only one input located at the tip element of the wing. A weak modal controllability means that the considered mode is difficult to excite by the chosen input. One may consider selecting an input location or several locations that would produce a relatively stronger modal controllability for the modes of interest. In our example shown above, it is seen that the dominant branch of the eigenvalues is the least controllable in comparison with other branches of eigenvalues regardless of the locations of the excitation inputs. Nevertheless, eigenvalues in the dominant branch are the most observable. In system identification, both controllability and observability are equally important. A combination of weak controllability and strong observability may be sufficient to identify 
the eigenvalues (modes) of interest. A conventional measure of the modal identifiability is the product of the modal controllability and observability for the mode of interest. An experimentalist before designing and performing an experiment, may need to use an analytical state-space model to evaluate the modal identifiability for the modes of interest in order to choose proper locations for excitation inputs and measurement outputs. In addition, the excitation signal must be rich in frequency for the modes of interest to excite these modes properly.

\section{Conclusions}

A state-space representation of a theoretical vortex lattice model has been developed using a system identification approach. The case of limited measurement data has been considered. This is done in anticipation of using the proposed system identification method with experimental data, e.g. a system identification model might be obtained from pressure (sensor) measurements on a wing in a wind tunnel. Two possible system identification approaches have been presented. Numerical results showed the importance of the choice of the wing motion or gust under consideration on building system identification models and predicting the vortex strengths or pressure on a wing. Modal controllability and observability have been discussed. Numerical results for the chosen delta wing vortex lattice model have shown that the modes corresponding to the dominant branch of the eigenvalues of the flow are the least controllable and most observable. By least controllable one means that these modes are difficult to excite by the type of downwash discussed in this paper such as the step angle of attack, frozen sharp edge gust, frozen gust of changing frequency, random downwash, or frozen random gust. On the other hand, by most observable one means that the dominant modes are easy to observe in terms of vortex strengths or the pressure distribution on the wing. Identifiability of modes depends clearly on both modal controllability and observability. The product of modal controllability and observability is commonly used as a measure of modal identifiability. 


\section{References}

1. Hall, K. C., "Eigenanalysis of Unsteady Flows About Airfoils, Cascades, and Wings," ALAA Journal, Vol. 32, No. 12, 1994, pp. 2426-2432.

2. Tang, D. M., Kholodar, D. B., Juang, J.-N., Dowell, E. H., "System Identification and POD Method Applied to Unsteady Aerodynamics," accepted for publication in $A L A A$ Journal, 2000.

3. Juang, J.-N., Applied System Identification, Prentice Hall, Inc., Englewood Cliffs, New Jersey 07632, 1994, ISBN 0-13-079211-X.

Table 1: Percent errors using the vortex strength data

\begin{tabular}{|c|c|c|c|c|c|}
\hline & $\begin{array}{c}\text { Step } \\
\text { Angle }\end{array}$ & $\begin{array}{c}\text { Sharp } \\
\text { Edge }\end{array}$ & $\begin{array}{c}\text { Swept } \\
\text { Gust }\end{array}$ & $\begin{array}{c}\text { Random } \\
\text { Gust }\end{array}$ & $\begin{array}{c}\text { Frozen } \\
\text { Random }\end{array}$ \\
\hline Step Angle, 67 & 0.41 & 3.40 & 246.07 & 84.11 & 79.09 \\
\hline Sharp Edge, 50 & 11.64 & 0.00 & 0.21 & 83.90 & 0.00 \\
\hline Swept Gust, 233 & 0.12 & 0.11 & 0.05 & 78.04 & 0.11 \\
\hline Random Gust, 300 & 0.00 & 0.00 & 0.00 & 0.00 & 0.00 \\
\hline Frozen Random, 104 & 13.49 & 0.07 & 0.25 & 89.57 & 0.07 \\
\hline
\end{tabular}

Table 2: Percent errors using the pressure data

\begin{tabular}{|c|c|c|c|c|c|}
\hline & $\begin{array}{c}\text { Step } \\
\text { Angle }\end{array}$ & $\begin{array}{c}\text { Sharp } \\
\text { Edge }\end{array}$ & $\begin{array}{c}\text { Swept } \\
\text { Gust }\end{array}$ & $\begin{array}{c}\text { Random } \\
\text { Gust }\end{array}$ & $\begin{array}{c}\text { Frozen } \\
\text { Random }\end{array}$ \\
\hline Step Angle, 17 & 0.05 & 4.25 & 168 & 83.8 & 90.7 \\
\hline Sharp Edge, 35 & 20.9 & 0.66 & 0.37 & 320 & 0.65 \\
\hline Swept Gust, 52 & 15.3 & 15.4 & 2.69 & 72.9 & 15.3 \\
\hline Random Gust, 110 & 0.42 & 0.42 & 0.07 & 0.39 & 0.42 \\
\hline Frozen Random,45 & 25.9 & 1.48 & 0.74 & 290 & 1.46 \\
\hline
\end{tabular}




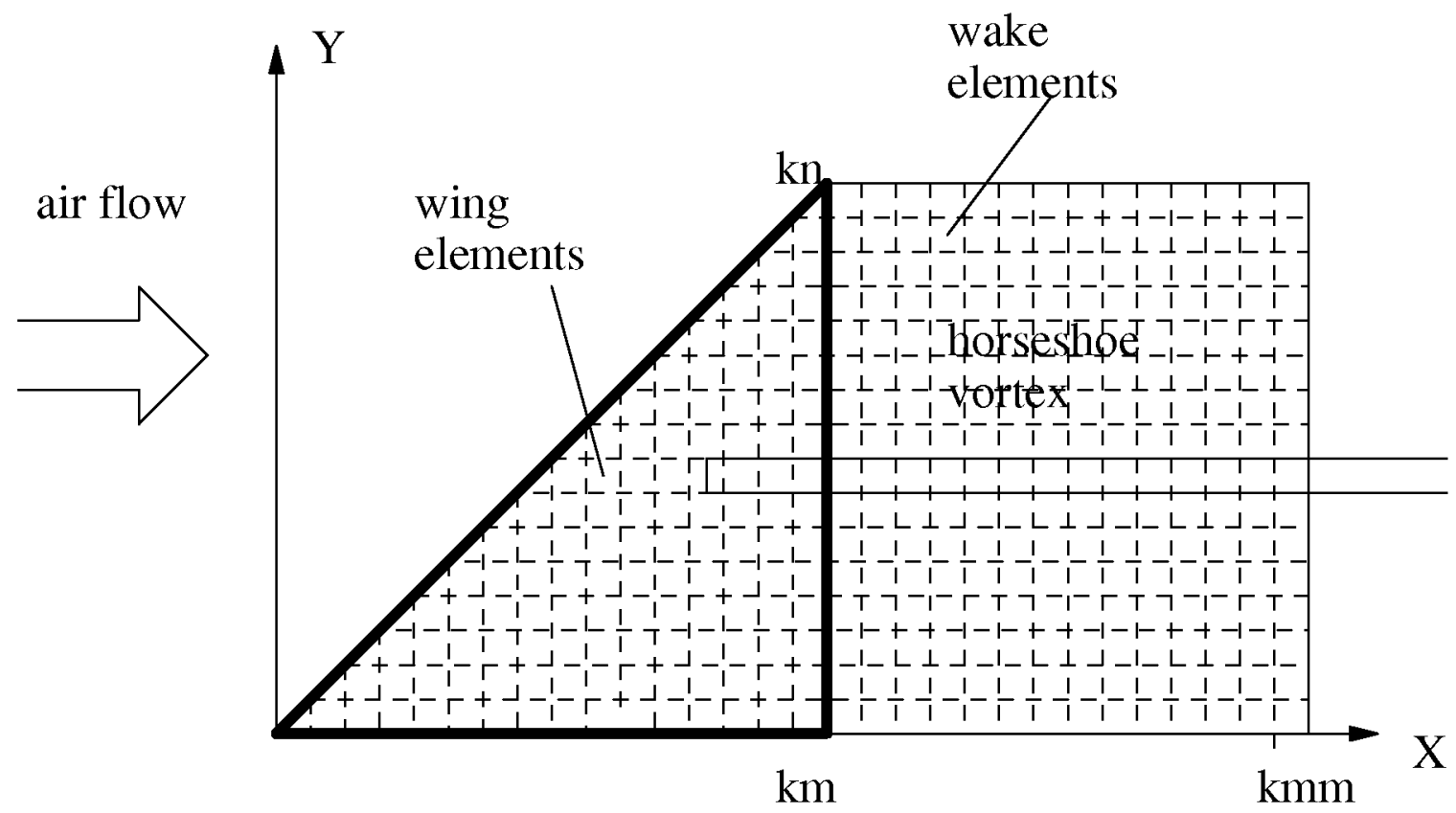

Figure 1: Vortex lattice model of unsteady flow about a delta wing. 

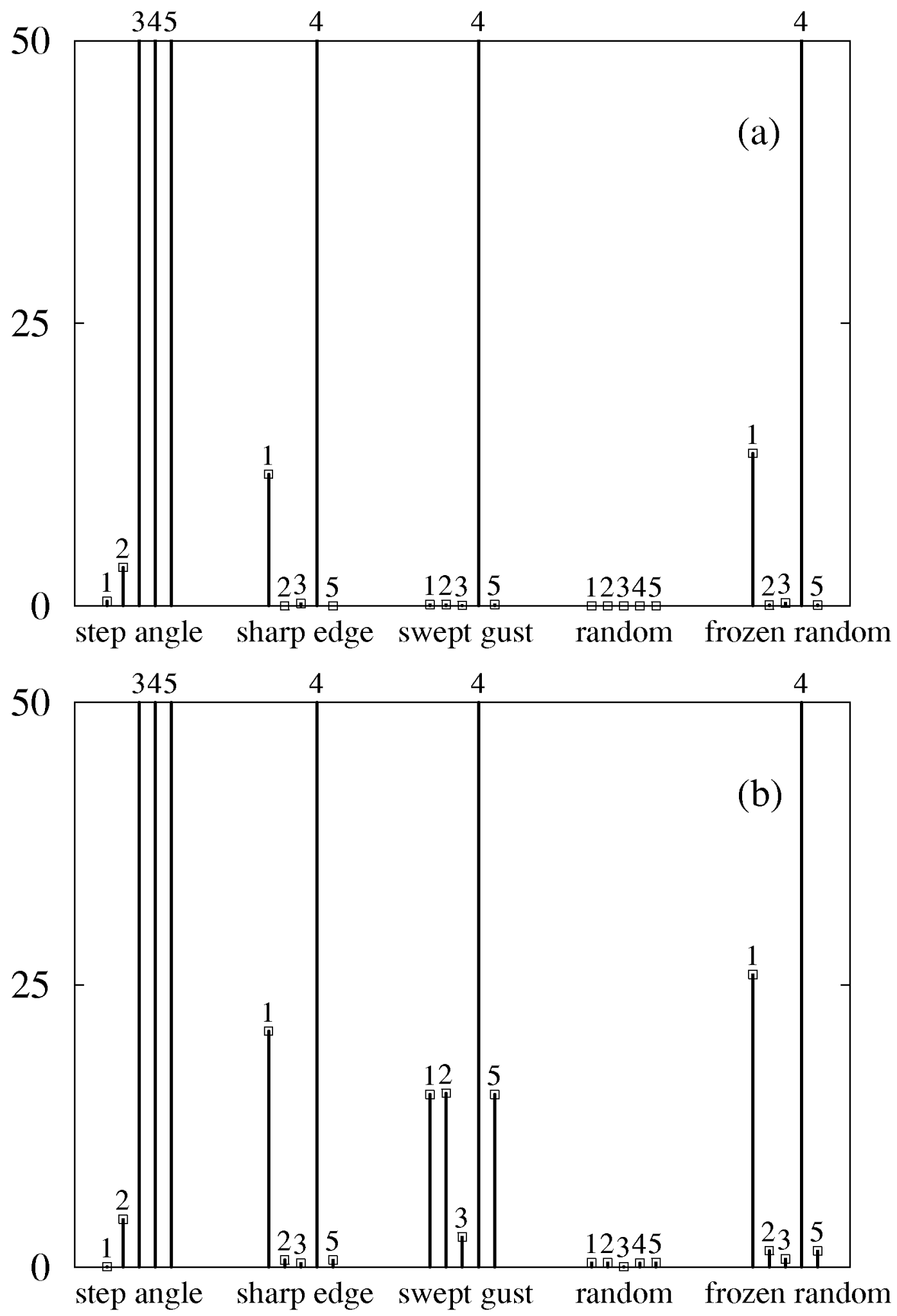

Figure 2: Percent error for (a) vortex strength and (b) pressure time histories for the five described excitations (marked by the numbers). The SID models used to simulate these histories are indicated along the horizontal axis. 

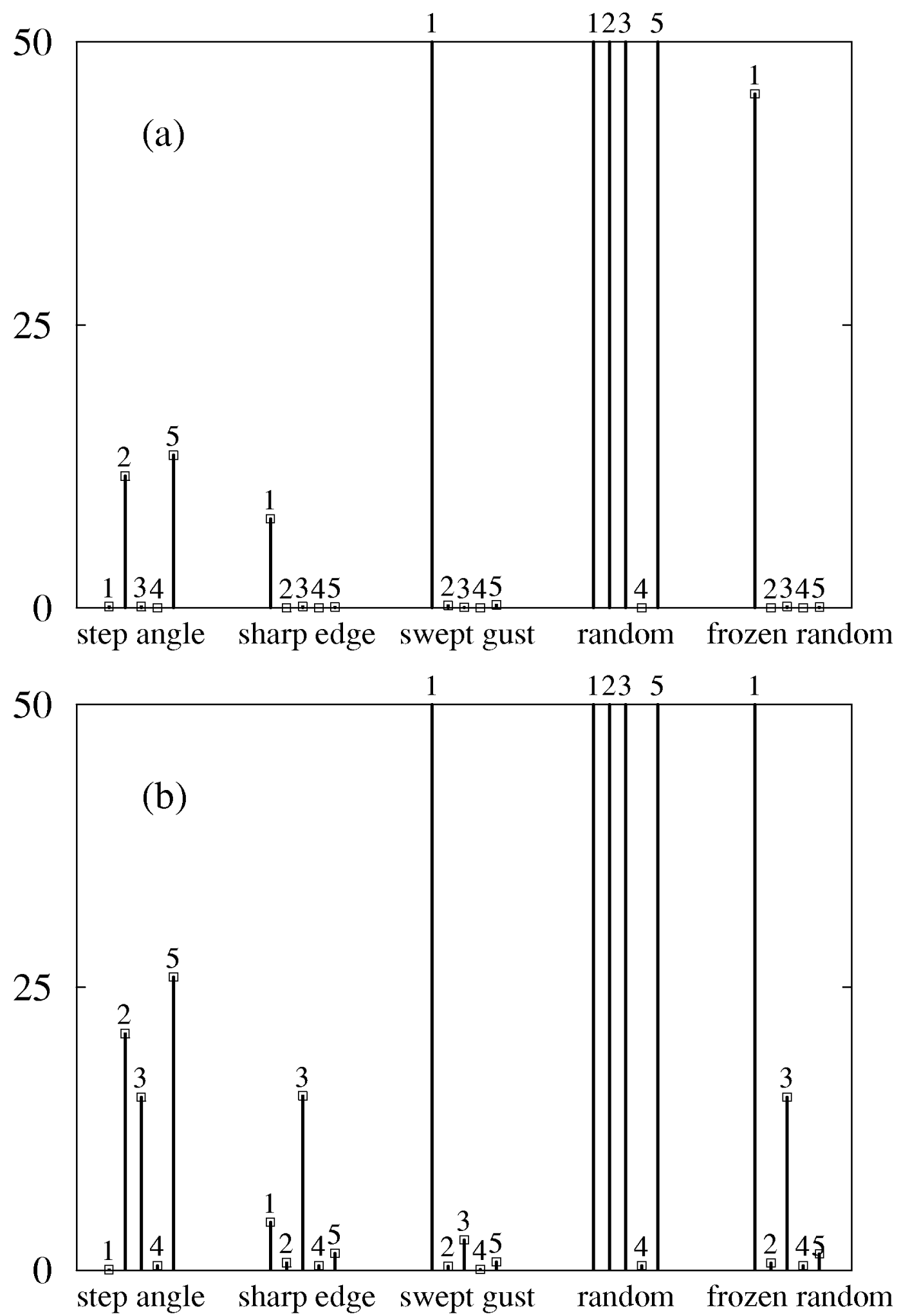

Figure 3: Percent error for (a) vortex strength and (b) pressure time histories that were simulated by the SID models (marked by the numbers). The flows under consideration are indicated along the horizontal axis. 

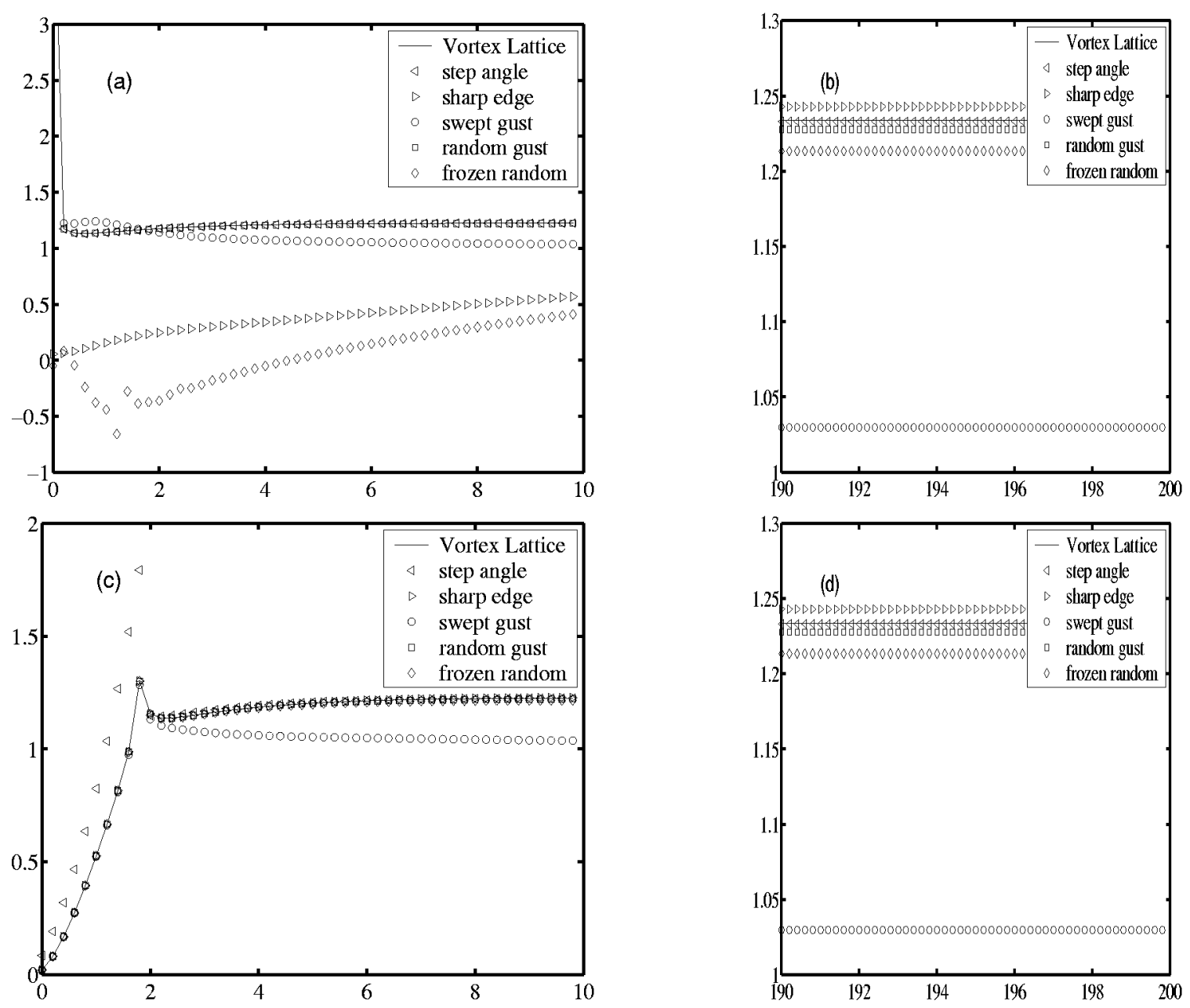

Figure 4: Lift time history for the excitations of ( $a$ and b) step angle of attack and (c and d) frozen sharp edge gust. 

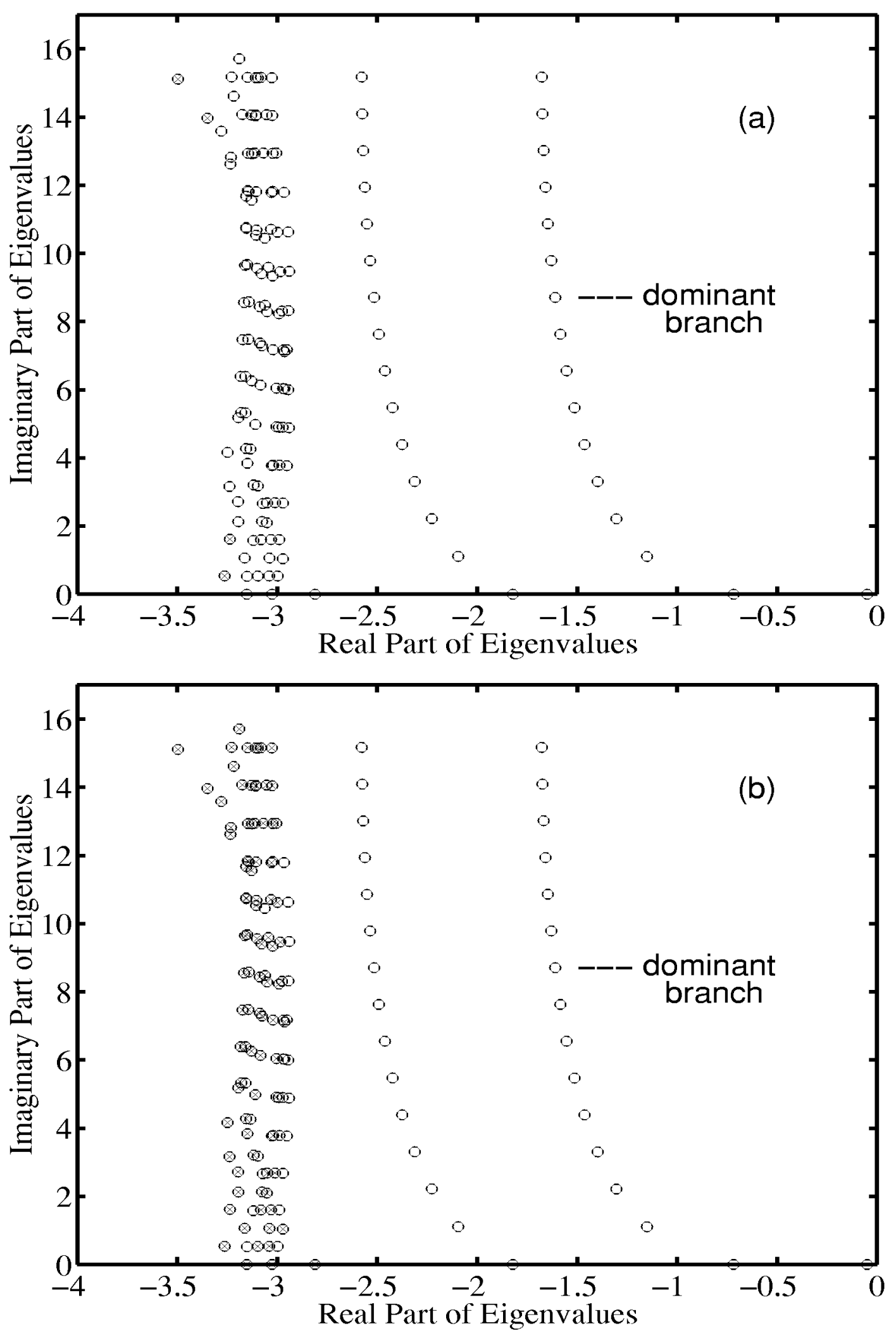

Figure 5: System eigenvalues. The crossed eigenvalues correspond to modes for which components in the scaled controllability vector are larger then (a) 0.1, (b) 0.01 . The largest controllability vector component is 1 (cf. Fig. 6a). 

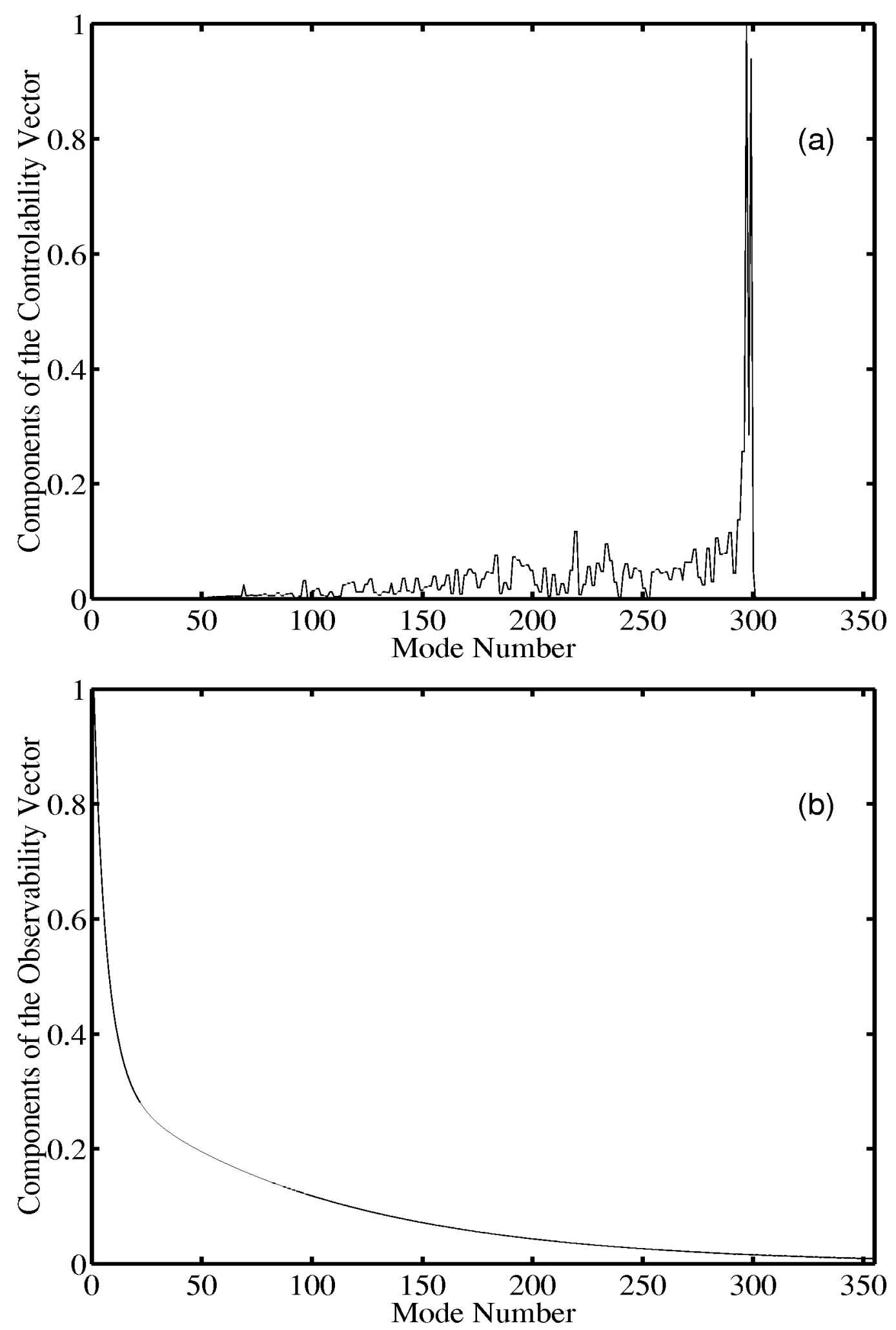

Figure 6: Contollability (a) and observability (b) vector components. In this case, the state, $A$, and input influence, $B$, matrices were derived in the close form from the VL model with the use of Eqs. (7), where the output influence matrix, C, is an identity matrix. The only input is located at the tip of the delta wing. 

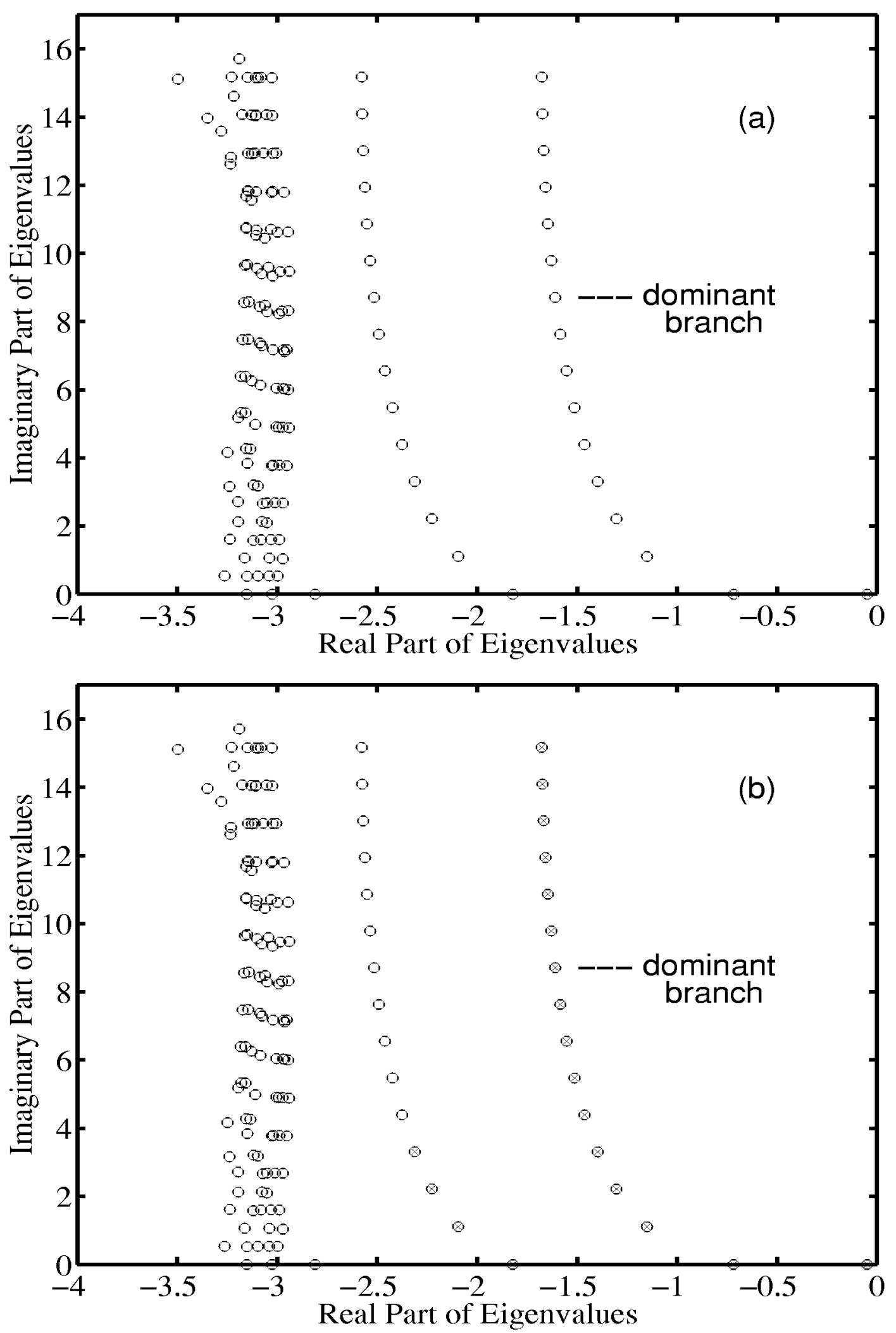

Figure 7: System eigenvalues. The crossed eigenvalues correspond to modes for which components in the scaled observability vector are larger then (a) 0.4, (b) 0.2 . The largest observability vector component is 1 (cf. Fig. 6b). 

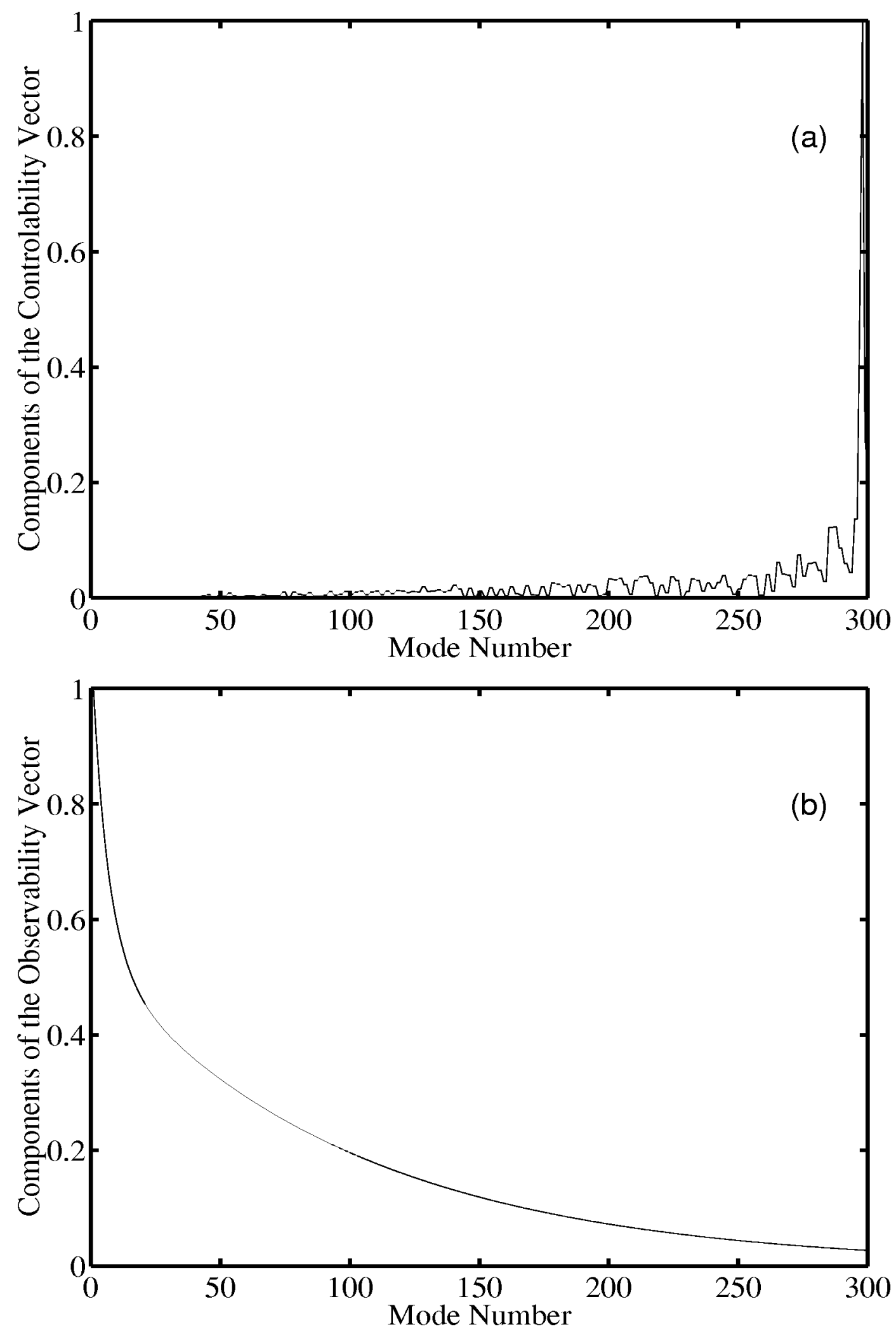

Figure 8: Contollability (a) and observability (b) vector components. In this case, matrices $\mathrm{A}, \mathrm{B}$, and $\mathrm{C}$ were identified from the vortex strength data when the wing was excited by the random gust. The only input is located at the tip of the delta wing. 

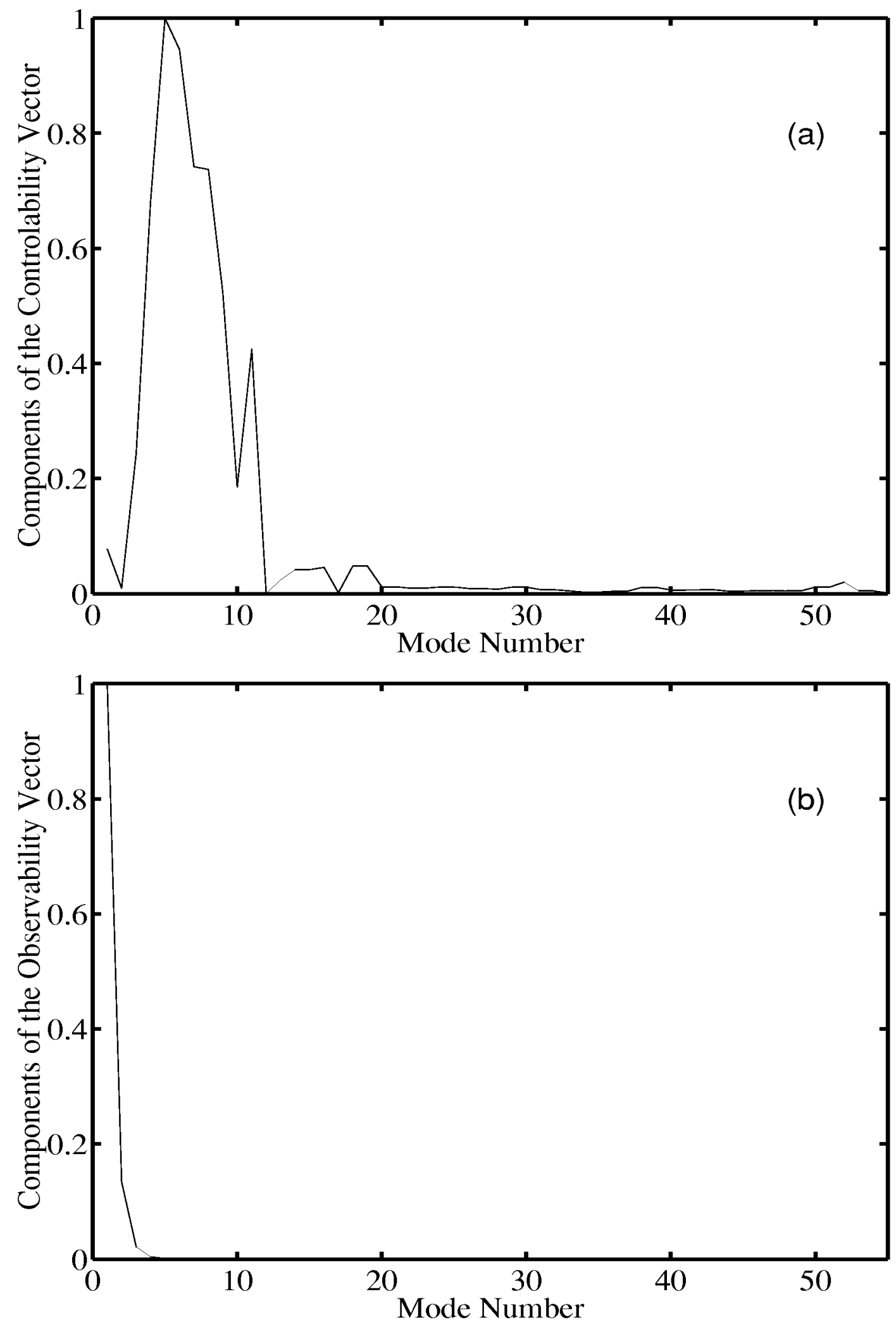

Figure 9: Contollability (a) and observability (b) vector components. In this case, matrices $\mathrm{A}, \mathrm{B}$, and $\mathrm{C}$ were identified from the pressure strength data when the wing was excited by the random gust. The only input is located at the tip of the delta wing. 
Public reporting burden for this collection of information is estimated to average 1 hour per response, including the time for reviewing instructions, searching existing data sources, gathering and maintaining the data needed, and completing and reviewing the collection of information. Send comments regarding this burden estimate or any other aspect of this collection of information, including suggestions for reducing this burden, to Washington Headquarters Services, Directorate for Information Operations and

Reports, 1215 Jefferson Davis Highway, Suite 1204, Arlington, VA 22202-4302, and to the Office of Management and Budget, Paperwork Reduction Project (0704-0188), Washington, DC 20503.

\begin{tabular}{|l|l|l}
\hline 1. AGENCY USE ONLY (Leave blank) & $\begin{array}{l}\text { 2. REPORT DATE } \\
\text { October } 2001\end{array}$ & $\begin{array}{l}\text { 3. REPORT TYPE AND DATES COVERED } \\
\text { Technical Memorandum }\end{array}$ \\
\hline
\end{tabular}

\section{TITLE AND SUBTITLE}

System Identification of a Vortex Lattice Aerodynamic Model

5. FUNDING NUMBERS

WU 706-32-21-01

\section{AUTHOR(S)}

Jer-Nan Juang, Denis Kholodar, and Earl H. Dowell

\section{PERFORMING ORGANIZATION NAME(S) AND ADDRESS(ES)}

8. PERFORMING ORGANIZATION

NASA Langley Research Center

Hampton, VA 23681-2199

National Aeronautics and Space Administration

Washington, DC 20546-0001

NASA/TM-2001-211229

\section{SUPPLEMENTARY NOTES}

12a. DISTRIBUTION/AVAILABILITY STATEMENT

12b. DISTRIBUTION CODE

Unclassified-Unlimited

Subject Category 39 Distribution: Nonstandard

Availability: NASA CASI (301) 621-0390

13. ABSTRACT (Maximum 200 words)

The state-space presentation of an aerodynamic vortex model is considered from a classical and system identification perspective. Using an aerodynamic vortex model as a numerical simulator of a wing tunnel experiment, both full state and limited state data or measurements are considered. Two possible approaches for system identification are presented and modal controllability and observability are also considered. The theory then is applied to the system identification of a flow over an aerodynamic delta wing and typical results are presented.

\begin{tabular}{|c|c|c|}
\hline \multicolumn{3}{|c|}{$\begin{array}{l}\text { 14. SUBJECT TERMS } \\
\text { System Identification; Vortex Lattice Model; Proper Orthogonal Decomposition; } \\
\text { Reduced Aerodynamic Model }\end{array}$} \\
\hline $\begin{array}{l}\text { 17. SECURITY CLASSIFICATION } \\
\text { OF REPORT } \\
\text { Unclassified }\end{array}$ & $\begin{array}{l}\text { 18. SECURITY CLASSIFICATION } \\
\text { OF THIS PAGE } \\
\text { Unclassified }\end{array}$ & $\begin{array}{l}\text { 19. SECURITY CLASSIFICATION } \\
\text { OF ABSTRACT } \\
\text { Unclassified }\end{array}$ \\
\hline
\end{tabular}

NSN 7540-01-280-5500
15. NUMBER OF PAGES 39

16. PRICE CODE A03

20. LIMITATION OF ABSTRACT

UL 